\title{
Accuracy of Elastography for Differentiation Benign and Malignant Breast Lesions
}

\author{
Aysar S Keiteb ${ }^{\mathbf{1}}$ and Shahad A Ibraheem*2 \\ ${ }^{1}$ Department of Radiological Techniques, College of Health and Medical Technologies, Iraq \\ ${ }^{2}$ Department of Imaging, Faculty of Medicine and Health Sciences, Malaysia
}

*Corresponding author: Shahad A Ibraheem, Department of Imaging, Faculty of Medicine and Health Sciences, Malaysia

\section{ARTICLE INFO}

Received: 慧 March 06, 2019

Published: 慧 March 20, 2019

Citation: Aysar S Keiteb, Shahad A Ibraheem. Accuracy of Elastography for Differentiation Benign and Malignant Breast Lesions. Biomed J Sci \& Tech Res 16(2)-2019. BJSTR. MS.ID.002814.

Keywords: Elastography; Ultrasound; Breast Cancer; Benign; Malignant; BIRADS

\section{ABSTRACT}

Introduction: Elastography is considered a non-invasive imaging methodology which decides the tumors concurring to their solidness. To survey the extra symptomatic esteem of elastography in combination with B-mode ultrasound in the characterization of solid breast lesions.

Purpose: To assess the role of elastography in characterizing breast masses and utilize of elastography to ultrasound for separate benign and malignant breast lesions.

Methods: The consider was conducted on 80 participants, each participant was subjected to complete history taking, exhaustive clinical examination. Females with a single undiagnosed solid breast lesion between March and May 2018. All patients had routine US and elastography utilizing GE Voluson E6 5-17 MHz linear probe.

Results: Among the 80 patients included in this study. 31 breast lesions were malignant \& 49 were benign. B-mode ultrasound was performed, and the lesions were categorized agreeing to the (BI-RADS) where chi-square statistical test uncovered that BI-RADS categories were essentially expanded among malignant cases $(\mathrm{P}<0.001)$. While the elastography classified concurring to altered Ueno and Ito elasticity score framework which benign lesions had elastography score 1, 2, 3 and 4 whereas malignant breast lesions had elastography score 4 and 5.

Conclusion: Elastography is a non-invasive imaging procedure which is done in the same session of ultrasound in an endeavor to extend and progress the precision of diagnostic effectiveness of ultrasound.

\section{Introduction}

Breast cancer is one of the foremost common cancers in women and its leading cause of cancer mortality in women and constitutes $14 \%$ of female cancer passing. It is expected that 41,070 passing due to BC happened within the year 2017 [1]. The expanding worldwide rate of malignant maladies has been recorded by World Health Organization (WHO) and is an issue of genuine concern, especially in creating countries where the increment appears to be more dominant [2]. Breast cancer has gotten to be a major danger to female wellbeing in Iraq, where it is the driving cause of passing after cardiovascular diseases among women, with a cancerrelated mortality rate of $23 \%$ [3]. It has been the highest-ranked malignancy among the Iraqi populace in common since 1986. The most recent Iraqi Cancer Registry uncovered that among an evaluated populace estimate of 32,500,000, an add up to of 21,101 unused cases of cancer were enrolled in 2012. Breast is reliable of diverse tissues counting fibrous, glandular as well. Breast is reliable of diverse tissues counting fibrous, glandular as well as fatty tissues $[4,5]$. The soft consistency of the breast is made of fatty tissues that encompass the breast glands.

Distinctive breast lesions are shown, fibroadenoma is considered the commonest benign tumor whereas the invasive ductal carcinoma is the commonest malignant tumor [6]. 
Therefore, elastography has as of late been presented to move forward the exactness and specificity of diagnostic ultrasound [7]. Ultrasound elastography is utilized to evaluate tissue stiffness. The procedure depends on the hypothesis that malignant and benign breast lesions have inalienable contrasts in solidness [7]. Distinctive ultrasound elastography procedures have been risen, counting compression strain imaging, and real-time shear velocity. These days compression ultrasound elastography is considered the foremost commonly utilized strategy in breast imaging [8]. A color outline is produced and is comparing to the gray-scale ultrasound images. A grading scale utilized to classify lesions, concurring to the color signature, has been presented by Youk et al. [9] (Table 1). The point of this pondering was to assess the diagnostic utility of sonoelastography in combination with ultrasonography in arranging to distinguish breast masses (benign from malignant).

Table 1: criteria of colure distribution in ELASTOGRAPHY color mode [9].

\begin{tabular}{|c|c|}
\hline Pattern Criteria of Colure Distribution & Result \\
\hline Homogenously light or dark blue & Benign \\
\hline $\begin{array}{c}\text { Heterogeneously, predominantly blue with spot-like green } \\
\text { or orange }\end{array}$ & Benign \\
\hline Heterogeneously, with patchy green, yellow or red & Malignant \\
\hline $\begin{array}{c}\text { Extremely heterogeneously, multicolor with red, orange, } \\
\text { green, blue and irregular areas without colors, which can } \\
\text { be named `multicolor sign }\end{array}$ & Malignant \\
\hline
\end{tabular}

\section{Patients and Methods}

Patients: This prospective study included 80 female patients who were alluded to the radiology department at Oncology teaching clinic, medical city, Baghdad, Iraq. The study was performed between March 2018 and May 2018 for assessment of not completely diagnosed solid breast lesions.

Inclusion criteria: Any female persistent who had a single solid breast lesion that was palpable by clinical examination or obvious on ordinary ultrasound and classified utilizing the Breast Imaging Reporting and Data System (BI-RADS) as categories II -V.

\section{Exclusion criteria:}

a) Patients with BI-RADS 0 and I categories, or with numerous lesions.

b) Patients with a histopathologically affirmed malignant breast mass, or who had already experienced ipsilateral breast surgery or gotten breast radiotherapy.

c) Pregnant and breastfeeding women

d) Women how used HRT

\section{Methods}

All patients were examined using B-mode ultrasound and elastography employing a 5-17- $\mathrm{MHz}$ linear transducer (GE Voluson E6) amid one examination sitting, by one radiologist with encounter more than 10 years in breast imaging. Both B mode and elastography has done by the same radiologist. The study convention was endorsed by the radiology department at health and technology college and informed consent was gotten from each subject included within the study. Each participant was subjected to complete history taking, and thorough clinical examination and they did the followings:

B-Mode Ultrasound: B-mode ultrasound was performed at first for all breast lesions. At that point, lesions were classified as agreeing to the BI-RADS category (Table 2) [10].

Table 2: BI-RADS category.

\begin{tabular}{|c|c|}
\hline Category & Management \\
\hline $\begin{array}{c}\text { Need additional imaging or } \\
\text { prior examination }\end{array}$ & $\begin{array}{c}\text { Recall for additional imaging and/or } \\
\text { await prior examination }\end{array}$ \\
\hline Negative & Routine screening \\
\hline Benign & Routine screening \\
\hline Probably benign & Tissue diagnosis \\
\hline Suspicious for malignancy & Tissue diagnosis \\
\hline Highly suggestive of malignancy & Management according to staging \\
\hline $\begin{array}{c}\text { Pathologically proven breast } \\
\text { cancer }\end{array}$ & \\
\hline
\end{tabular}

Elastography: Elastography was conducted employing a superficial transducer, which was connected opposite to the longest diameter of the breast lesion, applying light pressure to avoid distortion of high elasticity. The elastography images were gotten in "one-shot scan" mode. Contact jelly was applied (inadequate sums to decrease artifacts) and patients were inquired to hold their breath for some seconds whereas they were scanned. Colour mode information, speed, proliferation, and tissue elasticity were analyzed for each lesion once, using the frozen picture. Engendering mode shows a design of lines representing the exactness and reliability of the procured shear wave information. The more parallel lines, the more exact information. The width between the lines is more prominent in stiff lesions than in solid soft tissue. This data is utilized to direct the distinguishing proof of a region of interest (ROI) on ranges with more parallel form lines [3]. Color mode employments four color patterns (depicted in Table 1). Patterns 1 and 2 are considered to represent a benign lesion, while patterns 3 and 4 are considered positive for malignant lesion [10]. The imaging results were compared with histopathological results.

\section{Statistical Analysis}

Information was examined using IBM SPSS Insights 25. Descriptive statistic was performed in a shape of number and rate for qualitative data. Chi-squared test $\left(\chi^{2}\right)$ and independent paired t-test were utilized to consider the significance of affiliation between elstosonography of breast lesion and histopathology. A p-value of $\leq 0.05$ was considered significant. 


\section{Results}

This study included 80 patients with palpable breast lumps. Their ages extended from 20 years to 70 years with a mean \pm SD age of $40 \pm 13.54$ years and the mean $\pm S D$, to begin with, the menstrual cycle was $12 \pm 1.09$ years. Table 4 appears sociodemographic variables distribution in connection to breast screening by ultrasound combined with elastography, most respondents are Muslim, Urban, married with kids on breastfeeding, most of these women did not utilize birth control pills, Most of the participant had no family history of breast cancer (66.3). All patients were assessed by grayscale ultrasound and sono-elastography examination, 80 cases had numerous diverse pathological lesions. Histopathologic examination uncovered that 49 lesions $(61.3 \%)$ were benign and 31 (38.8\%) were malignant (Table 3), this table appears there's no significant association between breast lesions (benign and malignant) with sociodemographic factors but in term of family history of $\mathrm{BC}$ and age with $\mathrm{p}<0.05$. Agreeing to the BI-RADS classification, 7 breast lesions (8.8\%) were BI-RADS 2, 42 lesions (52.5\%) were BI-RADS 3, 8 lesions (10\%) were BI-RADS 4 and 23 lesions $(28.7 \%)$ were BIRADS 5. The connection between the BIRADS of the examined lesions and the diagnosis are summarized in Table 4. This table appears significant affiliation with a p-value $<0.05$. Elastography was performed in 80 cases and being classified agreeing to modified Ueno and Ito elasticity score system. Benign lesions had elastography score 1, 2, 3 and 4 as follow: 7 lesions (14.3\%) had elastography score 1, 9 lesions (18.4\%) had elastography score 2, 17lesions (34.7\%) had elastography score 3 and 16 lesions (32.7\%) had elastography score 4. However malignant breast lesions had elastography score 4 and 5 as follow: 12 injuries (38.7\%) had elastography score 4, 19 injuries (61.3\%) had elastography score 5 (Table 5).

Table 3: The relation between breast lesions and sociodemographic variables.

\begin{tabular}{|c|c|c|c|c|}
\hline \multirow[t]{2}{*}{ Variables } & \multirow[t]{2}{*}{ Total (no. \%) } & \multicolumn{2}{|c|}{ Breast Lesions } & \multirow[t]{2}{*}{$P$ value } \\
\hline & & Benign (no. \%) & Malignant (no. \%) & \\
\hline & & $49(61.3 \%)$ & $31(38.8 \%)$ & \\
\hline Age groups & & & & 0.18 \\
\hline $20-29$ & $17(21.3)$ & $13(76.5)$ & $4(23.5)$ & \\
\hline $30-39$ & $27(33.8)$ & $17(63.0)$ & $10(37)$ & \\
\hline $40-49$ & $15(18.8)$ & $10(66.7)$ & $5(33.3)$ & \\
\hline $50-59$ & $10(12.5)$ & $3(30.0)$ & $7(70)$ & \\
\hline $60-70$ & 11(13.8) & $6(54.5)$ & $5(5.5)$ & \\
\hline Religion & & & & 0.37 \\
\hline Muslim & $72(90)$ & $45(62.5)$ & $27(37.5)$ & \\
\hline Christian & $8(10)$ & $4(50)$ & $27(37.5)$ & \\
\hline Area & & & & 0.29 \\
\hline Urban & $68(85)$ & $43(63.2)$ & $25(36.8)$ & \\
\hline Rural & $12(15)$ & $6(50)$ & $6(50)$ & \\
\hline Marital Status & & & & 0.93 \\
\hline Single & $19(23.8)$ & $12(63.2)$ & $7(36.8)$ & \\
\hline Married & $55(68.8)$ & $33(60)$ & $22(40)$ & \\
\hline Divorce/Widow & $6(7.5)$ & $4(66.7)$ & $2(33.3)$ & \\
\hline Have Kids & & & & 0.34 \\
\hline Yes & $55(68.8)$ & $35(63.6)$ & $20(36.4)$ & \\
\hline No & $25(31.3)$ & $14(56)$ & $11(44)$ & 0.16 \\
\hline \multicolumn{5}{|l|}{ Breast feeds } \\
\hline Yes & $64(80)$ & $37(57.8)$ & $27(42.2)$ & \\
\hline No & $16(20)$ & $12(75)$ & $4(25)$ & 0.11 \\
\hline \multicolumn{5}{|l|}{ Birth control pills } \\
\hline Yes & $23(28.7)$ & $17(73.9)$ & $6(26.1)$ & \\
\hline No & $57(71.3)$ & $32(56.1)$ & $25(43.9)$ & \\
\hline The family history of BC & & & & $0.001^{*}$ \\
\hline Yes & $27(33.7)$ & $5(36.4)$ & $22(63.6)$ & \\
\hline No & $53(66.3)$ & $44(83)$ & $9(17)$ & \\
\hline
\end{tabular}




\begin{tabular}{|c|c|c|c|}
\hline Mean \pm SD years & $40 \pm 13.54$ & $38 \pm 13.0$ & $44 \pm 13.7$ \\
Age & $12 \pm 1.09$ & $12 \pm 1.12$ & $0.04^{*}$ \\
Menstrual Cycle & & 0.43 \\
\hline
\end{tabular}

Table 4: The relation between BI-RADS of the studies lesions and the diagnosis.

\begin{tabular}{|c|c|c|c|c|c|c|c|}
\hline \multirow{2}{*}{ Birad Grades } & \multicolumn{2}{|c|}{ Total (80) } & \multicolumn{2}{c|}{ Benign } & \multicolumn{2}{c|}{ Malignant } & \multirow{2}{*}{ P value } \\
\cline { 2 - 7 } & No. & \% & No. & \% & No. & \% & \\
\hline 0 & 0 & 0.0 & 0 & 0 & 0 & 0 & $0.001^{*}$ \\
\hline 1 & 0 & 0.0 & 0 & 0 & 0 & 0.0 & \\
\hline 2 & 7 & 8.8 & 7 & 14.3 & 0 & 0.0 & \\
\hline 3 & 43 & 52.5 & 42 & 85.7 & 0 & 0.0 & \\
\hline 4 & 8 & 10 & 0 & 0 & 8 & 25.8 & \\
\hline 5 & 23 & 28.7 & 0 & 0 & 23 & 74.2 & \\
\hline 6 & 0 & 0.0 & 0 & 0 & 0 & 0.0 & \\
\hline
\end{tabular}

\begin{tabular}{|c|c|}
\hline & 4 \\
\hline 3 & 1 \\
\hline & 3 \\
\hline 4 & 4 \\
\hline 5 & 4 \\
\hline & 4 \\
\hline
\end{tabular}

a) Lesions with BI-RADS 2 had elastography score 1, 2, 3 and 4.

b) Lesions with BI-RADS 3 had score 1, 2, 3 and 4 .

Table 5: The relation between elastography score and the diagnosis of breast lesions.

\begin{tabular}{|c|c|c|c|c|c|c|c|}
\hline \multirow{2}{*}{$\begin{array}{c}\text { Elastography } \\
\text { Score }\end{array}$} & \multicolumn{2}{|c|}{ Total (80) } & \multicolumn{2}{c|}{ Benign } & \multicolumn{2}{c|}{ Malignant } & \multirow{2}{*}{$\begin{array}{c}\text { P } \\
\text { value }\end{array}$} \\
\cline { 2 - 7 } & No. & \% & No. & \% & No. & \% & \\
\hline 1 & 7 & 8.8 & 7 & 14.3 & 0 & 0.0 & $0.001^{*}$ \\
\hline 2 & 9 & 11.3 & 9 & 18.4 & 0 & 0.0 & \\
\hline 3 & 17 & 21.3 & 17 & 34.7 & 0 & 0.0 & \\
\hline 4 & 28 & 35 & 16 & 32.7 & 12 & 38.7 & \\
\hline 5 & 19 & 23.8 & 0 & 0.0 & 19 & 61.3 & \\
\hline
\end{tabular}

This table appears noteworthy affiliation between elastography score with the diagnosis of breast lesions with a $\mathrm{P}$ value $<0.05$. None of the malignant lesions had score 1 or 2 , whereas none of the benign lesions had score 5 .

As regards the connection between BI-RADS classification and elastography score (Table 6):

Table 6: Classification of the lesions according to the BI-RADS and elastography score.

\begin{tabular}{|c|c|}
\hline Birads & Elastography Score \\
\hline 2 & 1 \\
\hline & 2 \\
\hline & 3 \\
\hline
\end{tabular}

c) Lesions with BI-RADS 4 had score 4 .

d) Lesions with BI-RADS 5 had score 4 and 5 .

e) Lesions with BI-RADS 6 had score 3. (These cases were diagnosed as breast cancer but we evaluate a de novo lesion after administration of the essential).

In this study: Fibroadenoma, simple cyst, and fibrocystic changes were the foremost common benign lesions whereas infiltrative ductal carcinoma was the foremost common malignant lesion. Fibroadenomas showed up smooth oval or adjusted in shape with well-defined edges, homogenous echotexture, isoechoic with bilateral acoustic shadowing, wider than taller and either softer than or had the same elasticity as adjoining glandular tissue with score 1 , 2 or 3 (Figure 1). Fibroadenomas sometimes have size and stiffness pattern by elastography which is similar to that of malignant lesions as in calcified fibroadenomas with elasticity score 3 or 4 (Figure 2). Malignant breast lesions are speculated, irregularly formed, unwell outlined, with heterogeneous echotexture, distorted design, central shadowing, taller than wider, small calcifications, elastography score 4 or 5 . They appeared larger on the elastography image due to higher visualization of the encircling desmoplastic reaction (Figures $3 \& 4$ ).

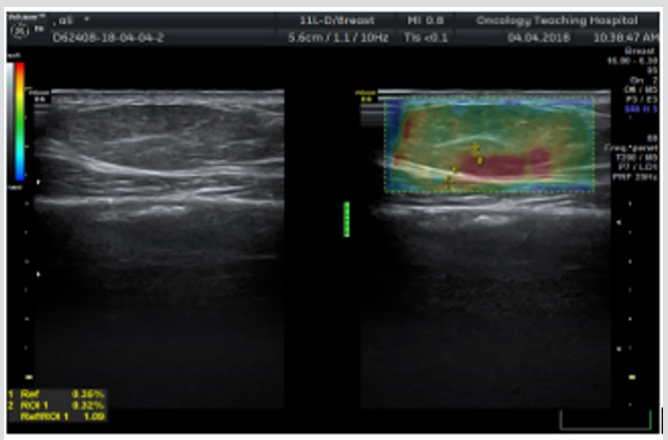

a) The conventional US showed well-defined iso to the hypoechoic focal lesion

b) On elastography: the lesion showed a heterogeneous but mostly green color signature with elastography score 2 . The diagnosis was confirmed by follow up after 6 months to be fibroadenomas.

Figure 1: 36 year's females with single left palpable breast lump. 


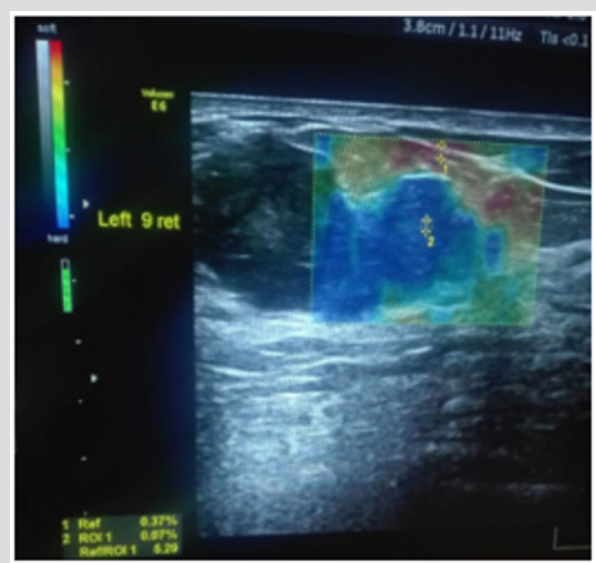

Figure 2: 23 years female with a palpable left breast lump, conventional US displayed hypoechoic lesion with irregular borders, posterior acoustic shadowing, elastography of the lesion revealed score 4 (the lesion was uniformly blue color signature confined to the visible margin of the lesion). The identification was confirmed infiltrating ductal carcinoma.

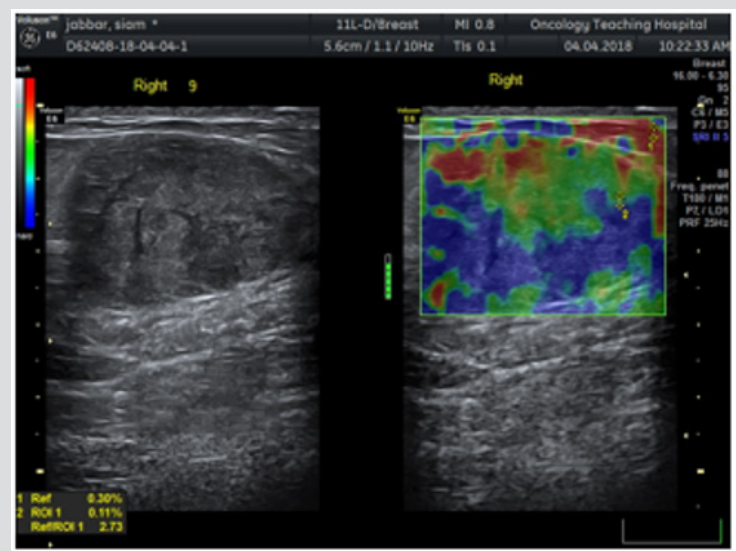

a) The conventional US showed well outlined hypoechoic focal lesion with multiple calcifications

b) On elastography: the lesion showed a heterogeneous however largely green and blue colors signature with elastography score four. The diagnosing was confirmed to be fibroadenomas.

Figure 3: 33 years female with a single right palpable breast lump.

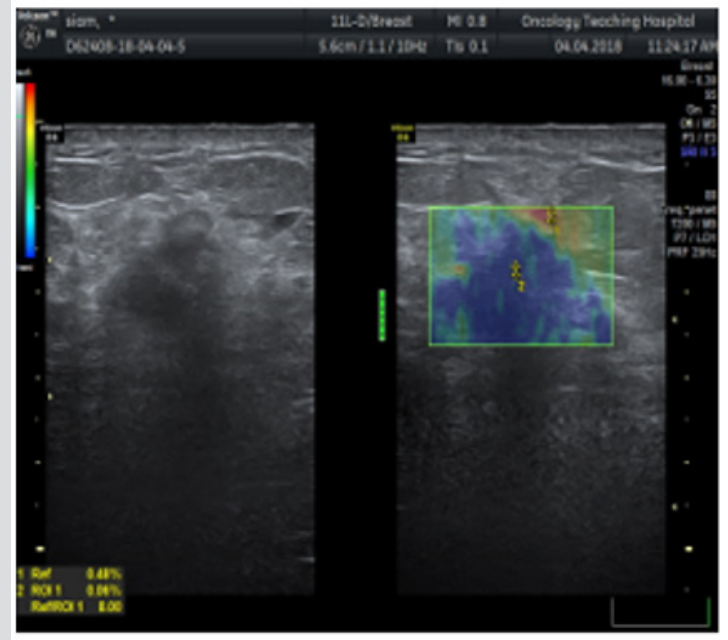

Figure 4: 33 years female with a palpable right breast lump, US and elastography, showed mass dense glandular tissue with score 5 (the lesion was blue color). The final identification was confirmed by core biopsy as ductal carcinoma in situ. 


\section{Discussion}

Over the years breast ultrasound elastography has evolved as an adjunct to the traditional US, changing into a valuable tool in clinical applications and currently there's competition with different imaging modalities like PET CT and magnetic resonance imaging $[11,12]$. ultrasound is the main imaging tool in young females. it's thought of as a sensitive modality to find breast cancers [13]. B-mode US depends primarily on morphologic criteria of breast lesions. A biopsy may be a necessary method to verify the identification of malignantlesions. but increased frequency of breast biopsies for benign lesions is taken into account another downside because of price, stress and redoubled risk of infection and don't forget it's still an invasive technique [14]. Our findings showed that breast screening during this group of women was comparable with the findings of the Iraqi Cancer Registry/Ministry of Health (20002009). The mean age was 42 years. The incidence in the age group (30-39) didn't decline since 2003; the incidence of all female breast cancer in Iraq (all ages) has up. However, breast cancer among Iraqi women still affects younger age groups than their counterparts in developed countries. any medical specialty analysis is required to look at doable causes and prevention measures [15]. Our result showed the urban ladies had a higher incidence than rural women this finding matched the study that done in Iraq among Kurdish women [16].

In this study 49 lesions (61.3\%) from 80 lesions were benign and 31 lesions (38.8\%) were malignant. consistent with BI-RADS analysis of the conventional B mode US, there have been 7 (14.3\%) lesions from 49 benign lesions had BI-RADS 2 and 42 (85.7\%) lesions had BI-RADS 3. whereas among 31 malignant breast lesions, $8(25.8 \%)$ lesions had BI-RADS 4 and 23 (75.2\% lesions had BI-RADS 5. The chi-square statistical test was unconcealed that BI-RADS categories were considerably increased among malignant cases (P $<0.001)$. This was in close conformity with results according to by Ikeda et al. who reported that B-mode US supported the characters of the BI-RADS had the sensitivity of $93.9 \%$, specificity of $88.3 \%$ and accuracy of $90.6 \%$ for all breast lesions [17]. The basic elasticity of biological tissue is altered by pathological processes. Since the important time elastography depicts useful tissue elasticity changes, its addition to B-mode US hyperbolic the performance in interpretation and final analysis of breast masses $[18,19]$.The interpretation criteria in elastography depend upon the qualitative parameter of the elasticity score [20]. Considering the elastography score on a complete range of 80lesions being classified consistent with changed Ueno and Ito elasticity score system. Among the 49 benign lesions in our study 7 Lesions had elastography score 1, 9lesions (18.4\%) had elastography score 2, 17 lesions (34.7\%) had elastography score 3 and 16 lesions (32.7\%) had elastography score 4 .

Among 32 malignant lesions in our study 12 lesions (38.7\%) had elastography score 4, 19 lesions (61.3\%) had elastography score 5. consistent with our study, considering the benign lesion with elasticity scores 1-3 and malignant lesion with elasticity scores $4-5$, our results were slightly totally different from the studies of Thomas et al. [21] and Navarro et al. [22]. These slight variations could also be attributed to the totally different incidence of breast cancer, different selection criteria of the patient in addition as a distinction within the variety of the studied cases and variations in the used elastography techniques. Our patients with simple breast cysts pictured the characteristic 3 layers pattern of blue-green-red colors (positive BGR sign) with blue color is being the superficial one whereas red color is that the deep one, with an es of one, even in massive dimension lesions. This pattern was explained to be an aliasing artifact [23]. Our results corroborate findings according to by previous studies of Booi RC et al. [23] US elastography will diagnose the simple breast cysts and differentiate it from complicated cysts consistent with the B mode findings additionally to elastography with high confidence so as to avoid biopsy in some case. Among benign lesions, fibroadenomas tumors represent the foremost common kind of solid breast mass.

At mammography, these lesions seem also outlined lobulated hypoechoic lesions with coarse benign popcorn calcification with the benign options because the lesion is wider than taller with its long axis parallel to the skin, but if fibroadenomas by $\mathrm{B}$ mode ultrasound have size options almost like malignant lesions elastography can facilitate in confirming the diagnosis of its benign nature. This is matching with a study conducted by Garra bs et al. [24] who proven that 73 of fibroadenomas may be diagnosed and differentiated type malignant lesions relying upon its size, stiffness, and brightness in ultrasound and elastography examination. Among 25 lesions of fibroadenomas, 9 lesions had elastography score 4 (false positive), this in agreement with studies as well as Giuseppetti gm et al. [25] WHO according that fibroadenomas sometimes have size and stiffness features in grayscale ultrasound and elastography that is a lot of or less almost like that of malignant lesions, nonetheless these false positive findings common to occur in fibroadenomas quite $2 \mathrm{~cm}$ with calcification. In our study ultrasound elastography of invasive ductal carcinoma unconcealed tougher lesions than benign and normal tissues with the larger size in elastography. This was according to some studies by Kamoi K et al. [26] that confirmed that elastography diagnoses the stiffness of the invasive ductal carcinoma and accurately diagnose its actual extension.

Also, sonoelastography is beneficial in diagnosis atypical carcinomas like the terribly little or hyperechoic ones or those related to acoustic improvement [26]. Invasive ductal carcinoma could categorically well outline rounded mass on ultrasound, however, this can be uncommon. In our study, 3 lesions from 17 malignant lesions were solid with areas of necrosis that show score with high strain, this was matching with studies together with Insana MF et al. [27] that reportable, tumor necrosis could provide 
false negative results with low strain index because it seems like a cyst. Some breast cancer could show benign options (score 1-3) on elasticity imaging like non-differentiated DCI, inflammatory carcinoma, hypercellular, necrotic or pseudocystic malignant lesion deep little neoplastic nodules and huge cancers over $2.5 \mathrm{~cm}$ in diameter [27]. Our study had some limitations. one observer study, that the interobserver agreement for the elasticity measuring couldn't be assessed, we have a tendency to assessed breast lesions with no thought of breast thickness, lesion depth or size, which can influence diagnostic performance. Therefore, a further study is required to verify the connection between the elasticity values and every variable and to spot the variables that contribute to false-negative or false-positive results. additionally, the results of B-mode ultrasound combined with elastography wasn't obtained by a radiologist however from statistical analysis of the results of the combined technique. Therefore, we've got not provided specific pointers for combining the BI-RADS categories with B-mode ultrasound and elastography options.

\section{Conclusion}

Combined B-mode ultrasound with shear-wave elastography can improve the general diagnostic show for the differentiation of benign and malignant breast lesion.

\section{References}

1. Siegel R, DeSantis C, Jemal A (2014) Colorectal cancer statistics 2017. CA: a cancer journal for clinicians 64(2): 104-117.

2. (2014) World Health Organization. WHO position paper on mammography screening. World Health Organization.

3. (2013) International Agency for Research on Cancer: Globocan 2012. Lyon, France, World Health Organization International Agency for Research on Cancer.

4. Alwan NA (2010) Breast cancer: demographic characteristics and clinicopathological presentation of patients in Iraq. East Mediterr Health J 16(11): 1159-1164.

5. (2010) Iraqi Cancer Board. Results of the Iraqi Cancer Registry 2008 Iraqi Cancer Registry Center, Ministry of Health, Baghdad, Iraq.

6. Tardivon A El CK, Thibault F, Wyler A, Barreau B, Neuenschwander S (2007) Elastography of the breast: a prospective study of 122 lesions. Journal de radiologie 88(5 Pt 1): 657-662.

7. Barr RG, Zhang Z (2014) Shear-wave elastography of the breast: value of a quality measure and comparison with strain elastography. Radiology 275(1): 45-53.

8. Ophir J, Alam SK, Garra B, Kallel F, Konofagou E, et al. (1999) Elastography: ultrasonic estimation and imaging of the elastic properties of tissues. Proceedings of the Institution of Mechanical Engineers, Part H: Journal of Engineering in Medicine 213(3): 203-233.

9. Youk JH, Gweon HM, Son EJ, Han KH, Kim JA (2013) Diagnostic value of commercially available shear-wave elastography for breast cancers: integration into BI-RADS classification with subcategories of category 4. European radiology 23(10): 2695-2704.

10. Lazarus E, Mainiero MB, Schepps B, Koelliker SL, Livingston LS (2006) BI-RADS lexicon for US and mammography: interobserver variability and positive predictive value. Radiology 239(2): 385-391.
11. Li G, Li DW, Fang YX, Song YJ, Deng ZJ, et al. (2013) Performance of shear wave elastography for differentiation of benign and malignant solid breast masses. PloS one 8(10): e76322.

12. Gheonea IA, Stoica Z, Bondari S (2011) Differential diagnosis of breast lesions using ultrasound elastography. The Indian journal of radiology \& imaging 21(4): 301-305.

13. Akhtar MS, Mansoor T, Basari R, Ahmad I (2013) Diagnoses of breast masses with ultrasonography and elastography: A comparative study. Clinical Cancer Investigation Journal 2(4): 311.

14. Costantini M, Belli P, Lombardi R, Franceschini G, Mulè A, et al. (2006) Characterization of solid breast masses: use of the sonographic breast imaging reporting and data system lexicon. Journal of Ultrasound in Medicine 25(5): 649-659.

15. Al Hashimi MM, Wang XJ (2014) Breast cancer in Iraq, incidence trends from 2000-2009. Asian Pacific journal of cancer prevention: APJCP 15(1): 281-286.

16. Majid RA, Hassan HA, Muhealdeen DN, Mohammed HA, Hughson MD (2017) Breast cancer in Iraq is associated with a unimodally distributed predominance of luminal type B over luminal type A surrogates from young to old age. BMC women's health 17(1): 27.

17. Ikeda K, Ogawa Y, Takii M, Sugano K, Ikeya T, et al. (2012) A role for elastography in the diagnosis of breast lesions by measuring the maximum fat lesion ratio (max-FLR) by tissue Doppler imaging. Breast Cancer 19(1): 71-76.

18. Kim MY, Cho N, Yi A, Koo HR, Yun BL, et al. (2013) Sonelastography in distinguishing benign from malignant complex breast mass and making the decision to biopsy. Korean journal of radiology 14(4): 559-567.

19. Thomas A, Degenhardt F, Farrokh A, Wojcinski S, Slowinski T, et al. (2010) Significant differentiation of focal breast lesions: calculation of strain ratio in breast sonoelastography. Academic radiology 17(5): 558563.

20. Sadigh G, Carlos RC, Neal CH, Wojcinski S, Dwamena BA (2013) Impact of breast mass size on accuracy of ultrasound elastography vs. conventional B-mode ultrasound: a meta-analysis of individual participants. European radiology 23(4): 1006-1014.

21. Thomas A, Fischer T, Frey H, Ohlinger R, Grunwald S, et al. (2006) Realtime elastography an advanced method of ultrasound: first results in 108 patients with breast lesions. Ultrasound in Obstetrics and Gynecology 28(3): 335-340.

22. Navarro B, Úbeda B, Vallespí M, Wolf C, Casas L, et al. (2011) Role of elastography in the assessment of breast lesions: preliminary results. Journal of Ultrasound in Medicine 30(3): 313-321.

23. Booi RC, Carson PL, O Donnell M, Roubidoux MA, Hall AL, et al. (2008) Characterization of cysts using differential correlation coefficient values from two dimensional breast elastography: preliminary study. Ultrasound in medicine \& biology 34(1): 12-21.

24. Garra BS, Cespedes EI, Ophir J, Spratt SR, Zuurbier RA, et al. (1997) Elastography of breast lesions: initial clinical results. Radiology 202(1): 79-86.

25. Giuseppetti GM, Martegani A, Di BC, Baldassarre S (2005) Elastosonography in the diagnosis of the nodular breast lesions: preliminary report. La Radiologia medica 110(1-2): 69-76.

26. Kamoi K, Okihara K, Ochiai A, Ukimura O, Mizutani Y, et al. (2008) The utility of transrectal real-time elastography in the diagnosis of prostate cancer. Ultrasound in medicine \& biology 34(7): 1025-1032.

27. Insana MF, Pellot Barakat C, Sridhar M, Lindfors KK (2004) Viscoelastic imaging of breast tumor microenvironment with ultrasound. Journal of mammary gland biology and neoplasia 9(4): 393-404. 
ISSN: 2574-1241

DOI: 10.26717/BJSTR.2019.16.002814

Shahad A Ibraheem. Biomed J Sci \& Tech Res

(C) This work is licensed under Creative

Submission Link: https://biomedres.us/submit-manuscript.php

\begin{tabular}{ll} 
BIOMEDICAL & \multicolumn{1}{c}{ Assets of Publishing with us } \\
RESEARCHES & - Global archiving of articles \\
\hline ISSN:2574-1241 & - Immediate, unrestricted online access \\
\end{tabular}

